\title{
Geldpolitik und Finanzkrise - Die Bedeutung nicht-konventioneller geldpolitischer Maßnahmen
}

\author{
Helene Schuberth
}

\begin{abstract}
Auf den Ausbruch der Finanzkrise im Sommer 2007 hat die Geldpolitik mit nicht-konventionellen Maßnahmen reagiert. Auch die Europäische Zentralbank (EZB) hat neben einer Senkung des Hauptrefinanzierungssatzes auf mittlerweile $1 \%$ auf solche Maßnahmen zurückgegriffen. Im ersten Jahr der Krise konzentrierten sich die unkonventionellen geldpolitischen Maßnahmen auf die Liquiditätsversorgung des Finanzsystems, was bis heute fortgesetzt und ausgebaut wurde. Diese reichen von Swap-Operationen in Devisen zur Bereitstellung von Fremdwährungsliquidität über die Ausweitung der refinanzierungsfähigen Sicherheiten bis zur unlimitierten Zuteilung von Liquidität zum jeweiligen Hauptrefinanzierungssatz im Rahmen des Tenders. Mit der Ankündigung des Erwerbs von Pfandbriefen im Ausmaß von 60 Mrd. $€$ folgt die EZB der Praxis der US-amerikanischen Zentralbank (Fed), die schon seit Längerem bestimmte Finanzmarktsegmente direkt mit Liquidität unterstützt. ${ }^{1}$
\end{abstract}

\section{1 \\ Einleitung}

In seiner Essay-Sammlung aus den 1980er Jahren „Can it Happen Again?" wie auch in seinem letzten großen Werk „Stabilizing an Unstable Economy" geht Hyman Minsky (1982, 1986) der Frage nach, ob sich die Depression der 1930er Jahre wiederholen könne, deren Ausmaß durch verheerende wirtschaftspolitische Fehler - von einer kontraktiven Geldpolitik, über restriktive Fiskalpolitik bis hin zu einer Beggar-thyneighbour-Politik in Form von Protektionismus im Handel und eines Abwertungswettlaufs - verstärkt wurde. Die Antwort war ein eindeutiges Nein. Die Wirtschaftspolitik habe, so Minsky, aus den Fehlern der Vergangenheit gelernt und sowohl Fiskal- als auch Geldpolitik würden in ähnlichen Situationen antizyklisch reagieren. Tatsächlich hat die Wirtschaftspolitik seit 2007 auf die Krise mit außergewöhnlichen Maßnahmen reagiert. Im vergangenen Herbst, als das Weltfinanzsystem am Rande des Zusammenbruchs stand, konnte der Kollaps von Banken, Versicherungen und anderen Finanzinstitutionen mit unabsehbaren realwirtschaftlichen Folgen letztlich durch eine einzigartige, international koordinierte Vorgehensweise von Staaten verhindert werden. Zu den Maßnahmen zählten die Rekapitalisierung von Banken, Garantien von Bankenemissionen, Haftungen von Bankeinlagen sowie allfällige Auffanglösungen. Die Fiskalpolitik ist weltweit expansiv - beim G20-Gipfel Anfang April wurde angekündigt, bis Ende 2010 fünf Billionen Dollar zur Bekämpfung der Krise auszugeben. ${ }^{2}$ Die Notenbanken haben bereits seit Ausbruch der Krise im August 2007 mit umfangreichen und außergewöhnlichen Maßnahmen reagiert; deren expansiver Impuls ist allerdings schwierig zu quantifizieren.

Dennoch ist heute der Einbruch wichtiger ökonomischer Variablen ähnlich dramatisch, wenn nicht sogar stärker, als unmittelbar nach 1929. Eichengreen/O'Rourke (2009) weisen darauf hin, dass die Industrieproduktion seit April 2008 weltweit ähnlich kräftig eingebrochen ist wie nach dem Juni 1929; der Welthandel und die Aktienkurse sogar noch drastischer. Ob möglichst bald ein Wendepunkt erreicht werden kann, hängt neben zahlreichen Faktoren auch davon ab, ob es gelingt, den nach wie vor beeinträchtigten Prozess der Kreditintermediation wieder funktionstüchtig zu machen.

Angesichts der bereits niedrigen Notenbankzinsen haben unkonventionelle Maßnahmen der Geldpolitik in diesem Zusammenhang in der Diskussion, aber auch in der Praxis, stark an Bedeutung gewonnen. Dabei handelt es sich um Maßnahmen, die bei einem Nullzinssatz, aber auch bei einem positiven Zinssatz gesetzt werden, um über die Steuerung des Notenbankzinssatzes hinausgehend expansive Impulse für die Realwirtschaft zu setzen. Der Einsatz nicht-konventioneller Instrumente der Geldpolitik hat zum Ziel, einer allfälligen Deflationsgefahr entgegenzuwirken und/oder eine zuvor dysfunktionale Liquiditätsversorgung des Banken- und Finanzsystems wiederherzustellen. $\mathrm{Zu}$ den nicht-konventionellen Mitteln der Geldpolitik zählen u. a. die Liquiditätsunterstützung des Bankensystems im Interesse der Finanzmarktstabilität, längerfristige Refi- nanzierungsoperationen, Ankauf von Hypothekaranleihen und privaten Schuldverschreibungen (Commercial Papers) sowie von Unternehmens- und Staatsanleihen durch die Notenbank.

Dieser Beitrag skizziert die Möglichkeiten der Geldpolitik bei Deflationsgefahr sowie dysfunktionalen Kreditmärkten und beeinträchtigtem Transmissionsmechanismus der Geldpolitik. Letzteres scheint in dieser Krise besonders relevant, wenngleich Ersteres als Möglichkeit nicht ausgeschlossen werden kann. Abschnitt 2 erörtert die ökonomischen Umstände, die den Einsatz nicht-konventioneller Maßnahmen der Geldpolitik sinnvoll erscheinen lassen. Abschnitt 3 kategorisiert die breite Palette von nicht-konventionellen Maßnahmen und diskutiert die möglichen Konsequenzen, die diese Maßnahmen für die Interaktion von Notenbank und anderen volkswirtschaftlichen Sektoren haben. Abschnitt 4 vergleicht die diesbezügliche Reaktion der EZB mit jener der Fed. Angesichts der Verlängerung der Notenbank-

\footnotetext{
Dieser Artikel gibt die Meinung der Autorin wieder, die nicht notwendigerweise mit jener der Oesterreichischen Nationalbank übereinstimmt.

2 Kommuniqué des G-20 Gipfels vom 2. April 2009, The Global Plan for Recovery and Reform. http:// www.londonsummit.gov.uk/resources/en/PDF/ final-communique.
}

Helene Schuberth, Dr., ist Senior Adviser in der Hauptabteilung Volkswirtschaft der Oesterreichischen Nationalbank. Arbeitsschwerpunkte: Geld- und Fiskalpolitik Finanzmarktregulierung, Europäische Integration. e-mail: Helene.Schuberth@oenb.at 
bilanzen, die einige der nicht-konventionellen Maßnahmen zur Folge haben, wird in der medialen und wirtschaftspolitischen Debatte gerne das Bild einer Liquiditätsschwemme oder des Anwerfens der Notenpresse bemüht. Abschnitt 5 diskutiert die Inflationsrisiken des Einsatzes nicht-konventioneller Mittel in der Geldpolitik. Der Beitrag schließt mit einem kurzem Resümee (Abschnitt 6).

\section{Unkonventionelle Maß- nahmen der Geldpolitik - Motivation}

Der Einsatz unkonventioneller Maßnahmen der Geldpolitik wird heute vor dem Hintergrund von zwei Aspekten diskutiert und praktiziert. Sie zielen erstens darauf ab, den im Zuge von Finanzkrisen häufig auftretenden Deflationsrisiken wirksam vorzubeugen (Gerlach 2009). Die konventionelle Geldpolitik als Teil der keynesianischen Nachfragesteuerung wird unwirksam, sobald sie die Nullzinsuntergrenze erreicht hat, die nicht unterschritten werden kann. Negative Nominalzinsen würden erreicht, wenn Gläubiger für das Verleihen von Geld eine Gebühr zahlen müssen. Dies gilt aufgrund des Umstands, dass die Geldhaltung eine Rendite von Null hat, als ausgeschlossen. Kein Sparer würde einen negativen Zins akzeptieren, sondern eher Bargeld horten, in das Finanztitel jederzeit konvertiert werden können. ${ }^{3}$

Ermittelt man den angemessenen Zinssatz mithilfe der Taylor-Regel, ergäbe sich angesichts der aktuell außergewöhnlich hohen negativen Produktionslücke, die allerdings auch nur mit Unsicherheit geschätzt werden kann, nominell ein negativer Zinssatz. ${ }^{4}$ Der Taylor-Zins gibt einen groben Anhaltspunkt für jenen Zinssatz, der die Produktions- und Inflationslücke schließt. Der Taylor-Zins muss jedoch mit großer Vorsicht interpretiert werden, da er sehr sensitiv auf die getroffenen Annahmen über die Höhe des realen Gleichgewichtszinssatzes, der gewählten Variable und der Reaktionskoeffizienten reagiert. Angesichts der Untergrenze von Null für den nominellen Zinssatz, kann die geldpolitische Ausrichtung nur durch nicht-konventionelle Maßnahmen weiter gelockert werden, um das Deflationsrisiko abzuschwächen. Wesentlich sind Maßnahmen der „quanti- tativen Lockerung", wie etwa der Kauf von Staats- und Unternehmensanleihen durch die Notenbank.

Zweitens können nicht-konventionelle Maßnahmen die durch die Finanzkrise beeinträchtigten Finanzierungsbedingungen in gewissen Marktsegmenten verbessern, indem beispielsweise versucht wird, Risikoprämien in bestimmten Märkten zu reduzieren. Ziel ist hier nicht primär die Stimulierung der wirtschaftlichen Aktivität, sondern die Vermeidung von Liquiditätsengpässen oder die Aktivierung von Handelsaktivitäten in verschiedenen Märkten. Letztlich soll auch die für die Umsetzung der Geldpolitik wichtige Funktionsweise der für den geldpolitischen Transmissionsmechanismus relevanten Finanzmärkte normalisiert werden. Im Unterschied zur konventionellen Geldpolitik, bei der Notenbanken den Banken temporär gegen Wertpapiere als Sicherheit Geld leihen, erwerben sie nun Wertpapiere auf direktem Weg (Bernanke 2009), um spezifische, etwa von Illiquidität betroffene Finanzmarktsegmente zu unterstützen. Diese als „,credit easing" zu bezeichnende Strategie zielt primär darauf $a b$, Liquidität zu erhöhen und Risikoprämien zu senken; sie ist daher auch bei positiven Leitzinsen praktikabel.

\subsection{AUSBREITUNG DER FINANZKRISE}

Noch im April 2007 kommt der Internationale Währungsfond (IWF) im Global Financial Stability Report (IMF 2007) zum Ergebnis, dass der zu diesem Zeitpunkt bereits seit einigen Monaten zu verzeichnende Einbruch der Preise am Immobilienmarkt in den USA sich vermutlich nicht wesentlich auf andere Finanzmarktsegmente übertragen wird. Im August 2007 wurden Finanzmarktakteure und Regulatoren dann doppelt überrascht: Die Ratingagenturen korrigierten ihre Bewertungen für hypothekenbesicherte Wertpapiere bzw. strukturierte Produkte gleich um mehrere Qualitätsstufen nach unten. Zudem wurde bekannt, dass große Mengen dieser Wertpapiere global von Finanzinstitutionen und auch von Zweckgesellschaften gehalten wurden. Die Herabstufung des Ratings strukturierter Produkte löste angesichts des hohen Kredithebels und des ungeahnten Ausmaßes von Fristentransformation der Zweckgesellschaften, deren Fremdkapitalquote regulatorisch unbeschränkt war, einen Flächenbrand aus. Dieser manifestierte sich zunächst in einem sich kaskaden- artig ausbreitenden Verfall von Vermögenswerten. Verstärkt wurde der Preisverfall durch die negative (prozyklische) Wirkung des Schuldenabbaus: Die Finanzinstitutionen, die in der Boomphase versucht hatten, ihren Kredithebel, gemessen an der Relation von Fremd- zu Eigenkapital, zu maximieren, reagierten nun mit dessen Reduktion (Deleveraging). Dies verstärkte den ohnehin bestehenden Verkaufsdruck von Vermögenswerten. Zahlreiche Institute, die über keine eigene Einlagebasis verfügten, wie die großen Investmentbanken oder Zweckgesellschaften, gerieten rasch in eine Situation der Illiquidität oder Insolvenz.

Die Lehman-Pleite am 15. September 2008 löste dann eine Kettenreaktion aus und wurde als Signal gedeutet, dass es in Zukunft nicht mehr in jedem Fall zu einer staatlichen Rettung (Bail-out) für Finanzinstitute kommen wird. Die damit einhergehende Unsicherheit über das Liquiditätsund Solvenzrisiko war so groß, dass nahezu das gesamte Kreditgeschäft zwischen den Banken, das ohnehin bereits seit Mitte 2007 erheblichen Störungen unterlag, zum Erliegen kam. Bereits zuvor manifestierte sich das große gegenseitige Misstrauen der Banken in einer zeitweise starken Verteuerung am Interbankenmarkt. Negative Auswirkungen auf die Realwirtschaft konnten aufgrund des entschiedenen Eingreifens von Notenbanken und Regierungen gedämpft, aber nicht verhindert werden. Die dramatische Zuspitzung der Krise im Herbst 2008 hat zu einer beinahe alle Sektoren und Regionen umfassenden Kontraktion des Wirtschaftsgeschehens geführt, von der wieder negative Rückkoppelungen auf die Bankbilanzen und die Finanzmärkte ausgegangen sind. Die krisenbedingte Verschlechterung des Finanzierungsumfelds hat die konjunkturelle Situation spürbar verschärft.

\subsection{FINANZKRISE UND TRANS- MISSIONSMECHANISMUS DER GELDPOLITIK}

Dabei haben die Turbulenzen am Geldmarkt einen Transmissionsmechanismus

3 Kürzlich ist allerdings eine heftige Debatte über die Bedingungen, die einen negativen Nominalzins ermöglichen, entbrannt (vgl. z. B. Buiter 2009).

4 Die Financial Times berichtete von einem internen Papier der Fed, in dem der Taylor-Zins für die USA auf $-5 \%$ geschätzt wird (http://www.ft.com/cms/ s/0/37877644-32c9-11de-8116-00144feabdc0. $\mathrm{html)}$ 
der Geldpolitik, den Zinskanal, wesentlich beeinträchtigt (IMF 2008). Notenbankzinssätze wirken nicht direkt auf die Kundenzinssätze der Banken, sondern beeinflussen in erster Linie die Geldmarktzinsen, die wiederum neben anderen Faktoren die Kundenzinssätze beeinflussen. Im Euroraum haben seit Beginn der Turbulenzen auf den internationalen Finanzmärkten die zunehmenden Unsicherheiten auf dem Interbankenmarkt die Differenz zwischen den Geldmarktsätzen und Notenbanksätzen temporär beträchtlich ansteigen lassen. Die Turbulenzen betrafen dabei nicht nur den Tagesgeldsatz, sondern zeigten sich auch am Geldmarkt mit längeren Laufzeiten, da auch aufgrund der Unsicherheit über die künftige Liquiditätssituation (Liquiditätsrisiko) sowie bezüglich der Kreditwürdigkeit der Gegenparteien (Kreditrisiko) die Nachfrage der Banken nach Krediten mit längerer Laufzeit auf dem Interbankenmarkt stieg. Zur temporär beobachteten Ausweitung der Differenz zwischen Geldmarkt- und Notenbankzinsen kam hinzu, dass sich die Differenz zwischen Geldmarktzins und Kundenzinsen zeitweilig ausweitete. In den letzten Monaten gingen allerdings die Kundenzinsen in der Regel mit den stark gefallenen Geldmarktzinsen zurück.

Die Kreditzinsen sind allerdings nur ein Aspekt der Finanzierungsbedingungen von Unternehmen und Haushalten. Zusätzlich spielt eine Reihe von indirekten Kosten der Kreditaufnahme eine Rolle, über die allerdings kaum Daten vorliegen. Hinweise auf deren Entwicklung können im Bank-Lending-Survey des Eurosystems gefunden werden, in dem die Banken u.a. nach ihrer Einschätzung einer Reihe von Kreditbedingungen gefragt werden. Dabei zeigt sich, dass die Banken seit Beginn der Finanzmarktkrise die Kreditnebenkosten kontinuierlich verschärft haben. Während die Kreditzinsen im Einklang mit dem sinkenden Hauptrefinanzierungssatz zurückgingen, wurden im ersten Quartal 2009 zum siebenten Mal in Folge die Bedingungen für die Vergabe von Krediten, wie die Sicherheitenerfordernisse, die Fristigkeit der vergebenen Kredite oder die Zusatzoder Nebenvereinbarungen, weiter verschärft, zuletzt aber weniger deutlich als in den Quartalen zuvor (EZB 2009). Auch die Margen für durchschnittliche und risikoreiche Kredite sind weiterhin angestiegen. Einige Banken geben auch an, unmittelbar das Kreditangebot zu beschränken. Die im
Bank-Lending-Survey angedeutete Verschärfung der Kreditbedingungen zeigt sich am Trendrückgang bei der Kreditvergabe im Euroraum. Die Jahreswachstumsrate der Kredite an Haushalte und Unternehmen befindet sich auf einem kontinuierlichen Abwärtstrend. In Kombination mit einer eingeschränkten Funktionsfähigkeit der Finanzierung über den Kapitalmarkt stellen die verschärften Kreditbedingungen eine Belastung für die Wirtschaft dar.

\subsection{FINANZKRISE UND DEFLATION}

Nach Finanzkrisen steigt das Deflationsrisiko. Der universelle Verfall von Vermögenspreisen, der dem Platzen einer spekulativen Blase folgt, führt dazu, dass Haushalte und Unternehmen versuchen, ihre Verschuldung zu reduzieren. Das Sparen steigt und die gesamtwirtschaftliche Nachfrage geht zurück. Da auch die Banken versuchen, ihre hohen Fremdkapitalquoten zu reduzieren, kommt es zusätzlich zur Einschränkung der Kreditvergabe.

Die besondere Aversion gegen Deflation liegt darin begründet, dass die Notenbank im Falle von Inflation die Zinsen unbegrenzt nach oben anheben kann; im Falle einer Deflation stößt sie allerdings rasch an die Null-Barriere, da die Nominalzinsen nicht unter Null fallen können. Es kommt zu steigenden Realzinsen, wenn ein zunehmendes Sinken des Preisniveaus erwartet wird. Im Zusammenhang von sinkenden Preisen und hoher Verschuldung sah Irving Fisher die treibende Kraft hinter den desaströsen realwirtschaftlichen Folgen des Börsencrash im Herbst 1929 (Fisher 1933). Geht die Gesamtnachfrage zurück, sinken die Preise immer stärker und die realen Kosten des Schuldendienstes erhöhen sich. Das erschwert nicht nur die Rückzahlung, sondern verstärkt auch den Druck, die Schulden noch weiter abzubauen und auch wichtige Investitionen nicht zu tätigen. $\mathrm{Zu}-$ sätzlich führt der in Erwartung sinkender Preise zu verzeichnende Rückgang der Ausgabenneigung zu weiteren Preisrückgängen. Weitergehende Deflationserwartungen steigern die reale Zinslast der Unternehmen und die Konsumzurückhaltung. Der Teufelskreis einer sich selbst verstärkenden Abwärtsspirale setzt ein.

Aus diesem Grund wird ein durch Nachfrageschocks ausgelöstes Deflationsrisiko als besonders bedrohlich angesehen. ${ }^{5}$ Das Erreichen der Null-Barriere bei den
Notenbankzinsen bedeutet aber nicht, dass der geldpolitische Spielraum bereits voll ausgenutzt ist, um einer Deflationsgefahr wirksam begegnen zu können. Das den Notenbanken zur Verfügung stehende Instrumentarium erlaubt es nicht nur, den kurzfristigen Zinssatz zu steuern, sondern auch andere Determinanten des für die wirtschaftlichen Entscheidungen relevanten Realzinssatzes, wie die Inflationserwartungen und die Risikoprämien. Japan, das Mitte der 1990er Jahre in eine Deflationsperiode eingetreten war, hat $2001 \mathrm{mit}$ dem Einsatz nicht-konventioneller Maßnahmen der Geldpolitik begonnen. Die Bank of Japan setzte zu Beginn am längerfristigen risikofreien Zinssatz an, der durch die Ankündigung weiterhin niedriger kurzfristiger Zinsen und später durch die Erhöhung von Guthaben, die die Geschäftsbanken bei der Bank of Japan halten (quantitative Lockerung), gesenkt wurde (Bordo/Filardo 2005).

\section{3 Maßnahmen der Geldpolitik}

\subsection{INSTRUMENTE IM ÜBERBLICK}

Der Blick in die USA, nach Großbritannien und in die Schweiz oder nach Schweden zeigt, dass dort die geldpolitischen Leitzinssätze zum Teil bereits unter $1 \%$ gesenkt wurden. Zinspolitischer Spielraum besteht dort also nicht mehr oder höchstens in einem sehr geringen Ausmaß. Trotzdem agiert die Geldpolitik, und sie verfügt noch über eine Fülle von Möglichkeiten, jenseits des engen Rahmens der Zinspolitik wirksam zu werden. Zum einen erfolgt das über eine starke Ausweitung der Notenbankbilanzen, indem den Geschäftsbanken (viel) mehr Liquidität als üblich direkt zur Verfügung gestellt wird. Zum anderen verändert sich gleichzeitig die Struktur der Notenbankbilanzen, weil andere Geschäfte als die übliche Bereitstellung von kurzfristiger Liquidität an das Bankensystem nun die Struktur der Geldpolitik prägen.

\footnotetext{
5 Ein Deflationsdruck hingegen, der im Zuge von Produktivitätszuwächsen oder Rückgängen der Importpreise entsteht, wird als Angebotsdeflation oder "benign deflation" bezeichnet (Borio/Filardo 2004)
} 
Welche Instrumente vorrangig zum Einsatz kommen, hängt von der Problemstellung sowie von der konkreten Ausgestaltung des operationalen Rahmenwerks der Geldpolitik ab, die im internationalen Vergleich unterschiedlich ist (Jobst 2009). Ist die Funktionsweise des Interbankenmarktes gestört und kommt es zu einem massiven Anstieg der Liquiditätspräferenz der Banken bzw. zur Verknappung der Liquidität im Bankensystem, so kann primär mit unkonventionellen Geldmarktmaßnahmen die Liquidität und damit die Funktionsfähigkeit wichtiger Finanzmärkte unterstützt werden. So kann die Fristigkeit der Liquiditätsoperationen auf eine längere Laufzeit ausgedehnt werden. Weiterhin kann die Definition der zentralbankfähigen Sicherheiten ausgeweitet werden, ebenso der Kreis der im Geschäft mit der Notenbank zugelassenen Gegenparteien.

Steht die Vermeidung eines Deflationsszenarios im Vordergrund, sind Kapitalmarktmaßnahmen, die auf die Senkung der längerfristigen Zinsen sowie den leichteren Zugang der Unternehmensfinanzierung abzielen, geeignete Instrumente. Durch den Kauf von Unternehmensanleihen sollte die Emissionstätigkeit stimuliert werden und deren Risikoprämie sinken, wodurch die Refinanzierung der Unternehmen erleichtert wird. Der Kauf von Staatsanleihen durch die Notenbank erfolgt mit dem Ziel, Risikoprämien und Zinssätze zu senken.

Einem Anstieg von Risikoprämien sowie einer angebotsseitigen Kreditverknappung kann sowohl mit den oben genannten Geldmarktmaßnahmen begegnet werden als auch mit solchen, die am Kapitalmarkt ansetzen.

Angesichts der Konjunkturstabilisierungsprogramme und der Kontraktion des Wirtschaftsgeschehens, die mit sinkenden Steuereinnahmen sowie steigenden Staatsausgaben verbunden sind, sowie der hohen budgetären Kosten von Banken-Rettungspaketen, die eine teilweise Übertragung der Risken von den Banken an den öffentlichen Sektor implizieren, ${ }^{6}$ wird der direkte Kauf von Staatsanleihen durch die Notenbank verstärkt diskutiert und in zahlreichen Fällen praktiziert. Primäres Ziel ist, den risikofreien langfristigen Zinssatz sowie die Risikoprämien von Staatsanleihen, die im $\mathrm{Zu}$ ge des Anstiegs der Staatsschuld zum Teil gestiegen sind, zu reduzieren. Die realwirtschaftlichen Wirkungen des Erwerbs von

\section{Abb. 1: Interaktion von Notenbank, Regierung, Banken, Haushalten und Unternehmen im nicht-konventionellen Regime}

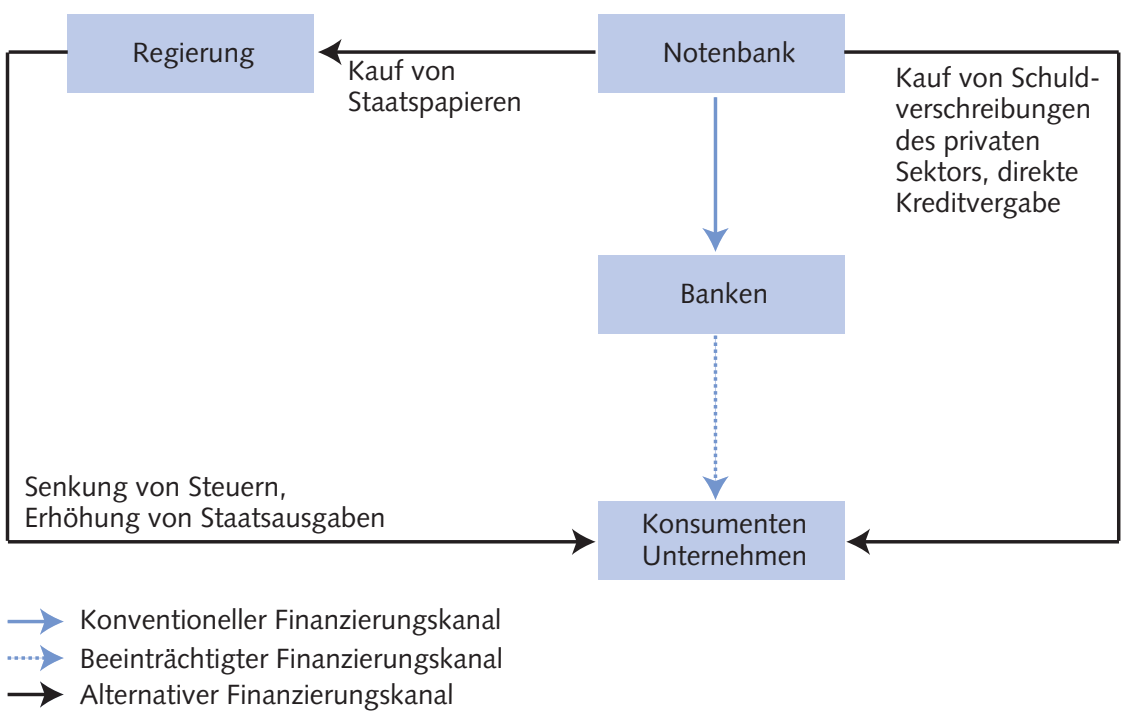

Quelle: Darstellung der Autorin.

WSI MITTEILUNGEN

Staatsschuldpapieren durch die Notenbanken lassen sich am besten durch drei Transmissionskanäle beschreiben. Zunächst sollten die Erwartungen in Richtung niedrigerer zukünftiger Zinssätze beeinflusst werden. Diese Signalwirkung, die hinsichtlich ihrer Effekte der glaubwürdigen Ankündigung eines niedrigen Zinspfades in der $\mathrm{Zu}$ kunft ähnlich ist, wird allerdings nur dann wirksam, wenn der Kauf von Staatsanleihen durch die Zentralbank nicht die Inflationserwartung erhöht. Zusätzlich zur Signalwirkung tritt ein Vermögenseffekt ein, indem das Vermögen der Besitzer von Staatsanleihen durch deren Kurszuwächse steigt. Wichtig ist zudem der Fiskalkanal in einer außergewöhnlichen Krisensituation (Bernanke et al. 2004). Sind fiskalische expansive Impulse zur Krisenbekämpfung begleitet vom Kauf von Staatsanleihen durch die Notenbank, so ist die (bei rationalen Erwartungen unterstellte) Befürchtung zukünftiger Steuererhöhungen zur Bedienung der gestiegenen Staatsschuld gedämpft. Bei einer Steuersenkung würde daher nicht nur, wie das Ricardianische Äquivalenztheorem unterstellt, das Sparen steigen, sondern auch der Konsum. Der von Vertretern dieses Theorems zugrunde gelegte Zusammenhang von expansiver Fiskalpolitik und der Erwartung steigender zukünftiger Steuerzahlungen würde durch monetäre Staatsfinanzierung außer Kraft gesetzt, die Wirksamkeit von Konjunkturstabilisierungsprogrammen erhöht. Ob diese Effekte tatsächlich eintreten, ist dann letztlich eine empirische Frage.

\subsection{INTERAKTION VOLKSWIRT- SCHAFTLICHER SEKTOREN BEI NICHT-KONVENTIONELLEN MABNAHMEN DER GELDPOLITIK}

In einem konventionellen Regime moderater Inflation, eines soliden Bankensystems und eines hohen Grades an Finanzmarktstabilität ist es das Ziel der Geldpolitik vieler Notenbanken, Preisstabilität zu gewährleisten sowie - in gewissem Ausmaß - Konjunkturschwankungen zu glätten (Svensson 1997) oder aber auch Wachstum und

6 Reinhart/Rogoff (2009) zeigen, dass im Durchschnitt vergangener Finanzkrisen drei Jahre nach Ausbruch der Krise die Staatsschuld real um $89 \%$ gestiegen ist.

$7 \quad$ Mit dem Übergang zur Inflationszielstrategie vieler Industrieländer in den frühen 1990er Jahren und auch zunehmend einiger Schwellenländer wurde Preisstabilität überwiegend als primäres Ziel der Geldpolitik statutarisch verankert. Auch der Vertrag von Maastricht legt die Sicherung der Preisstabilität als das primäre geldpolitische Ziel des Europäischen Systems der Zentralbanken (ESZB) fest, verpflichtet jedoch auch zur Verwirklichung der Ziele der Gemeinschaft, die in Artikel 2 EG-Vertrag festgelegt sind, wie beispielsweise eine hohe Beschäftigung. Der Humphrey Hawkings Akt aus dem Jahr 1978 legt hingegen fest, dass die Förderung des Wirtschaftswachstums neben der Sicherung der Preisstabilität ein gleichrangiges geldpolitisches Ziel der US-amerikanischen Fed ist (Judd/ Rudebusch 1999) 


\section{Abb. 2: Federal Reserve Bank - Struktur Aktiva}

- in Mrd. US Dollar -

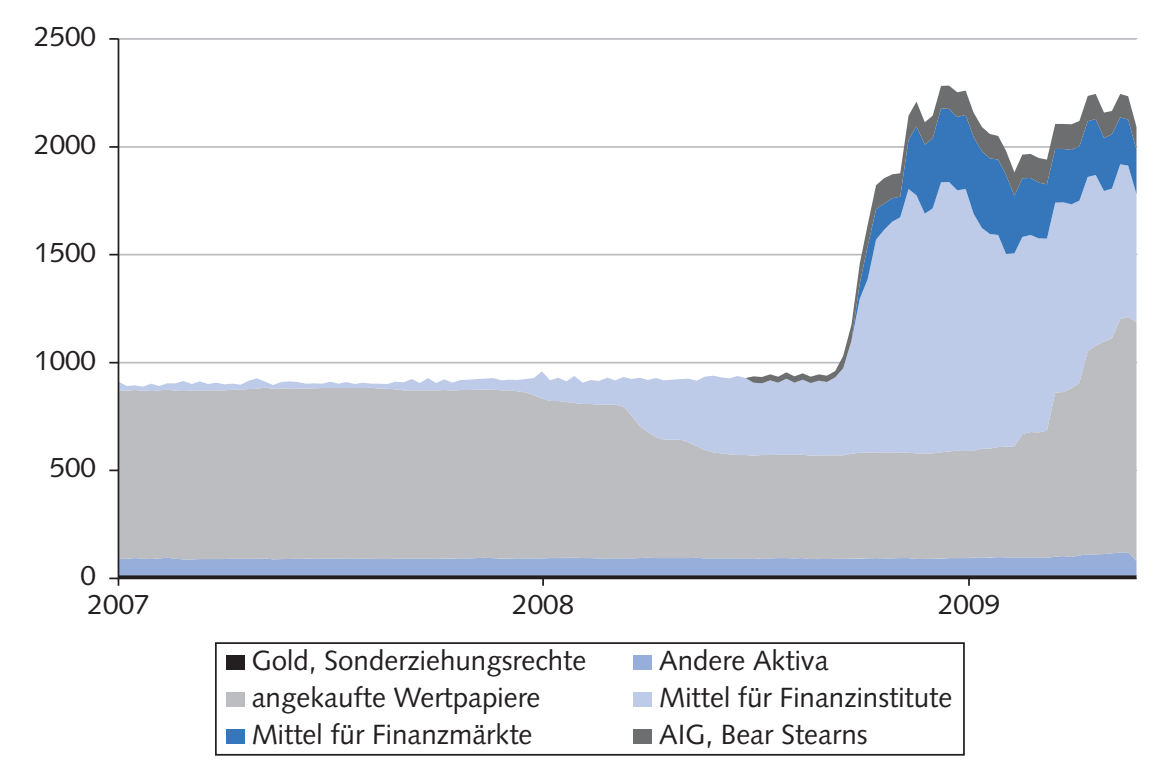

Quelle: Federal Reserve Bank.

WSI MITTELUNGEN

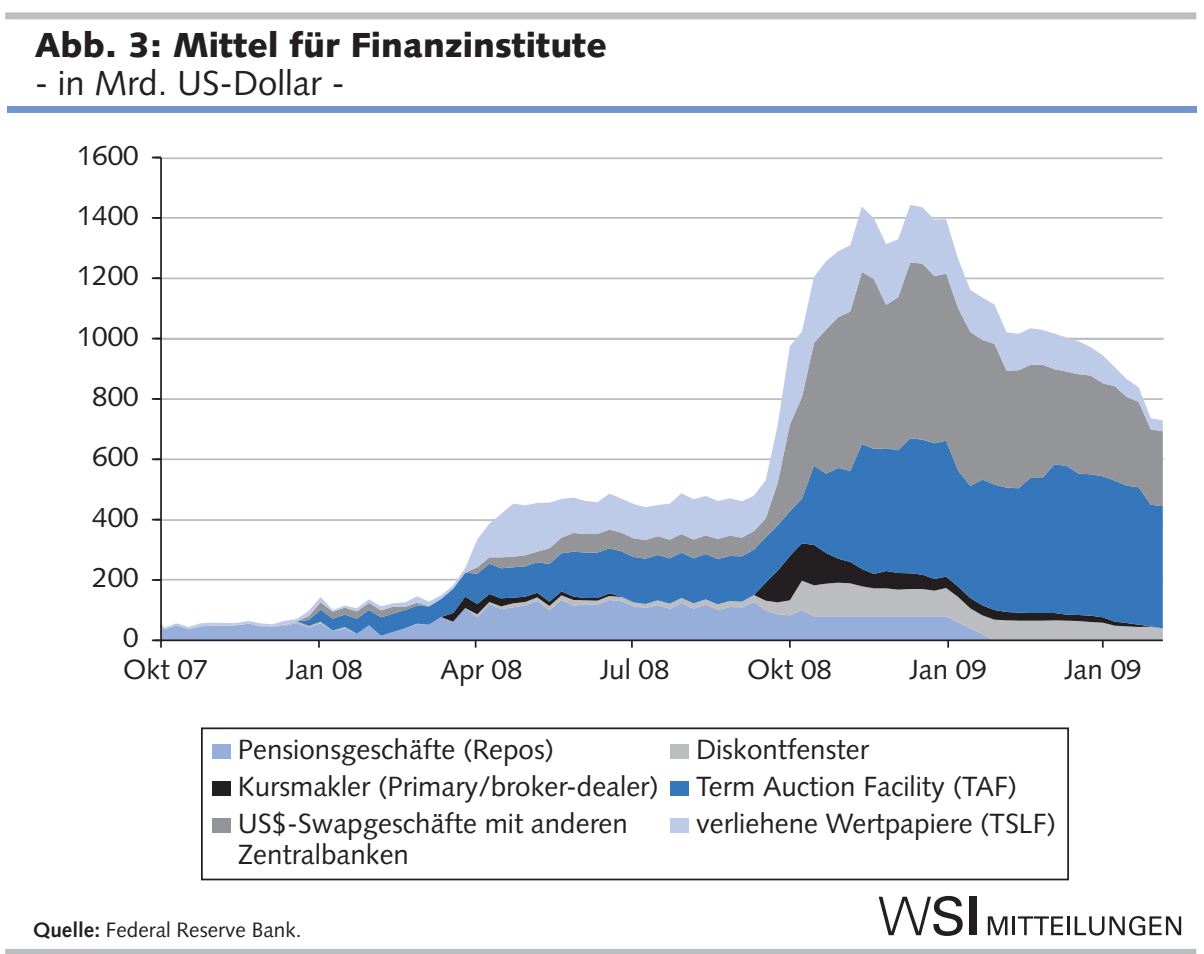

Beschäftigung sicherzustellen. ${ }^{7}$ In einem nicht-konventionellen Regime, in dem die Zinssätze die Nullgrenze annähernd erreicht haben, die Intermediatisierungsfunktion der Banken und der Finanzmärkte gestört und das Deflationsrisiko hoch ist, tritt neben die Ziele des konventionellen Regimes das Ziel der Stabilisierung des Finanzsystems. Stabile Finanzmärkte sind die Basis für einen effizienten Transmissionsmechanismus der Geldpolitik. Zudem ermit bankenbasierter Finanzierungsstruktur dar, wenn die traditionellen Finanzierungsströme nicht hinreichend funktionieren.

Um den in Finanzkrisen üblichen (schmerzhaften) Schuldenabbau des privaten Sektors zu kompensieren und damit dessen realwirtschaftliche Auswirkungen zu dämpfen, kann der Staat seine Schulden erhöhen. Die Staatsschuld steigt nicht nur infolge von Bankenrettungs- und Konjunkturpaketen, sondern wesentlich im Zuge des Wachstumseinbruchs (Reinhart/ Rogoff 2009). Der Fiskalstimulus erfolgt dabei über Steuersenkungen (Bernanke 1999) und/oder über eine Erhöhung des öffentlichen Konsums und der Investitionen (Krugman 2005). Eine Reihe von alternativen Finanzierungskanälen kompensiert dabei den beeinträchtigten Finanzierungskanal von den Banken zu den Unternehmen und Haushalten. Die Notenbank kann entscheiden, direkt private und öffentliche Schuldtitel zu erwerben. Unter diesen außergewöhnlichen Umständen ist eine Koordination von Geld- und Fiskalpolitik hilfreich, die mit der Unabhängigkeit von Notenbanken durchaus vereinbar ist - so, wie die Verfolgung eines gemeinsamen Zieles zweier unabhängiger Staaten die jeweilige nationale Souveränität nicht beeinträchtigt (Bernanke 2003).

\section{$\angle$}

\section{Nicht-konventionelle Maßnahmen - Vergleich USA und Euroraum}

\subsection{USA}

In Reaktion auf den Ausbruch der Krise im Sommer 2007 ergriff die Fed eine Reihe von Maßnahmen, die zunächst darauf ausgerichtet waren, das geldpolitische Instrumentarium zu adaptieren, um den Finanzsektor angesichts des dysfunktionalen Geldmarktes besser mit Liquidität zu versorgen. ${ }^{8}$ So wurde beispielsweise der zuvor enge Kreis der Banken, der Zugang zur Notenbankfinanzierung hatte, erweitert. $A b$ bildung 2 zeigt deutlich, dass bis Herbst 2008 keine Verlängerung des Umfangs der Bilanz der Fed stattgefunden hat, allerdings hat sich deren Zusammensetzung geändert. So wurde über diverse Instrumente die Liquiditätszufuhr an die Finanzinstitute ausgeweitet, allerdings wurden im selben Umfang Wertpapiere verkauft. Weiterhin wurden Staatsschuldtitel gegen weniger li-
8 Ein Überblick über die von der Fed neu eingerichteten Instrumente und Programme findet sich unter: http://www.federalreserve.gov/monetarypolicy/bst.htm. 
quide Wertpapiere ausgeliehen, um die Liquidität zu erhöhen. Auch diese Maßnahme war neutral im Hinblick auf das Bilanzvolumen. Erst ab Herbst 2008 kam es rasch zu einer starken Ausweitung der Bilanzsumme, die seither etwa auf diesem Niveau verblieben ist.

Die von der Fed gewählten Maßnahmen lassen sich anhand von drei Gruppen von Instrumenten erklären. Eine erste Instrumentengruppe setzt direkt an der traditionellen Funktion von Notenbanken an, Finanzinstituten kurzfristige Liquidität bereitzustellen (Abbildung 3). Unter dem Gesichtspunkt der Stabilität des Finanzsystems erfolgt das aber nun in einem viel größeren Ausmaß über neue Instrumente und auch an neue Empfänger, die von der Liquiditätsknappheit auf den Finanzmärkten in besonderer Weise betroffen sind. Die Bilanzverlängerung der Fed seit Herbst 2008 ist wesentlich auf diese Instrumentengruppe zurückzuführen. So wurde im Dezember 2007 die Term Auction Facility (TAF) eingerichtet, durch die eine sehr große Gruppe von Banken direkten $\mathrm{Zu}$ gang zur Notenbankliquidität bekam und eine breitere Palette von Sicherheiten verwendbar wurde. Die TAF wurde seit Herbst 2008 stark ausgeweitet. Durch die Term Securities Lending Facility (TSLF) können Banken temporär illiquide Wertpapiere gegen liquide Staatstitel tauschen, die dann im Rahmen von Rückkaufgeschäften (Repos) der Liquiditätsbeschaffung dienen. Diese Fazilität war zuletzt rückläufig. $\mathrm{Zu}$ erwähnen sind auch die insbesondere seit Herbst 2008 stark in Anspruch genommenen Devisen-Swap-Linien mit anderen Notenbanken, durch die die Verfügbarkeit von Dollar-Liquidität außerhalb der USA verbessert werden sollte.

Mittels einer zweiten Gruppe von Instrumenten versorgt die Fed seit Herbst 2008 direkt Schuldner und Investoren mit Liquidität, die für das Funktionieren von bestimmten Marktsegmenten, die ab Herbst 2008 massiv unter Druck gerieten, von entscheidender Bedeutung sind ( $A b$ bildung 4). Sie reichen von Fazilitäten für Geldmarktfonds über Commercial-PaperProgramme bis hin zu Krediten von Kreditkartenfirmen oder an Kleinunternehmen. Dieser Teil der Maßnahmen verbindet gleichermaßen Elemente der Finanzmarktstabilisierung wie auch der Finanzierung. $\mathrm{Zu}$ erwähnen sind die Asset-Backed Commercial Paper Money Market Mutual Fund Liquidity Facility (AMLF), die Commer-



cial Paper Funding Facility (CPFF), die Money Market Investor Funding Facility (MMIFF) sowie die Term Asset-Backed Securities Loan Facility (TALF). Das Volumen dieses Programms für Auto-, Konsum- und Studentenkredite ist noch sehr klein.

Der dritte Bereich von unkonventionellen Maßnahmen des Federal Reserve greift direkt in zentrale Kreditmärkte der

US-Wirtschaft ein. Das erfolgt über den direkten Ankauf von Wertpapieren staatlicher Agenturen in der Wohnbau- und der Unternehmensfinanzierung. Zielsetzung dieser Instrumente ist, die Zinssätze in diesen wichtigen Finanzierungsbereichen zu senken und einen entsprechenden Konjunkturimpuls zu setzen. Abbildung 5 zeigt, dass die Fed ab Anfang 2008, im Zuge der Ausweitung der direkten Liqui-






\section{Abb. 6: Konsolidierte Bilanz des Eurosystems - Aktiva}

- in Mrd. Euro -

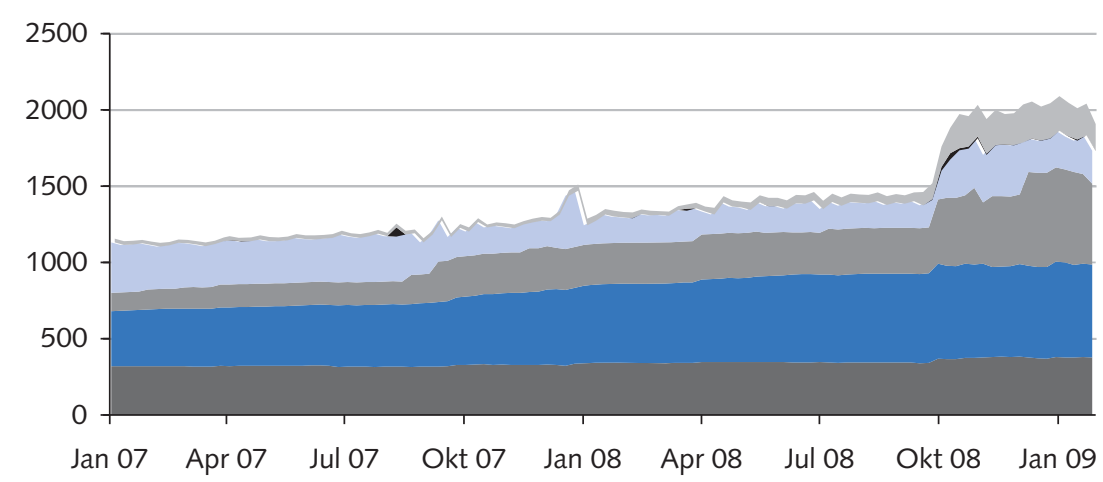

Ansprüche in Fremdwährungen gg. Euroraum

- Kreditfazilität und Feinsteuerungsoperationen

Hauptrefinanzierung

langfristige Refinanzierung

Andere Aktiva

Gold und Fremdwährung

Quelle: Europäische Zentralbank.

Abb. 7: Konsolidierte Bilanz des Eurosystems - Verbindlichkeiten - in Mrd. Euro -

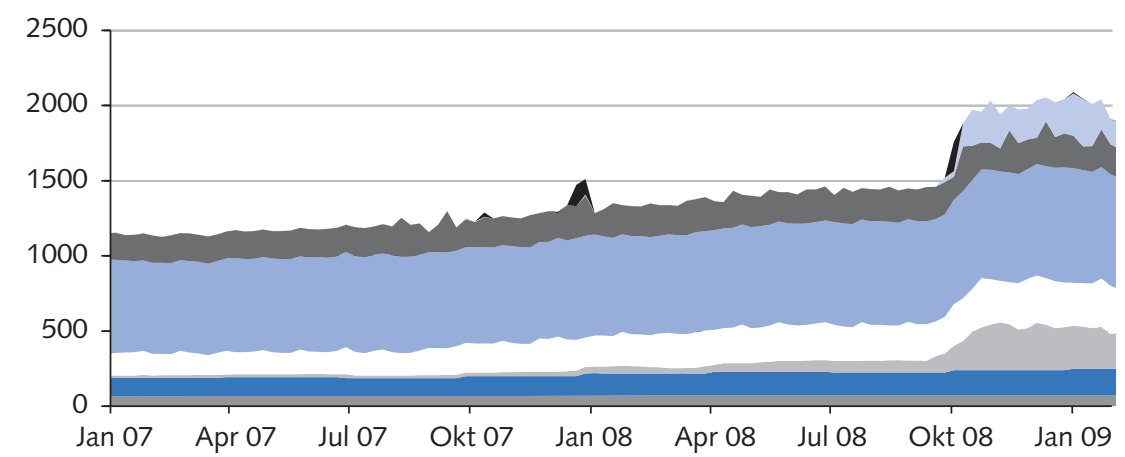

sonstige liquiditätsabschöpfende Operationen

Einlagefazilität

- Einlagen auf Girokonten

Banknoten im Umlauf

$\square$ Sonstige Verbindlichkeiten

Verbindlichkeiten gg. Ausland in Euro

- Ausgleichsposten aus Neubewertung

- Kapital und Reserven

ditätsbereitstellung an Banken, den Umfang ihres Portfolios an Staatspapieren vorübergehend deutlich verringert hatte, insbesondere den der kurzfristigen Wertpapiere (treasury bills). Im März 2009 kündigte die Fed an, den Ankauf von Staatspapieren wieder aufzunehmen. Im Februar 2009 begann sie zudem mit dem Erwerb von verbrieften Hypothekarkrediten, ein Programm, das seither stark ausgeweitet wurde.
WSI MITTELUNGEN

WSI MITTELLUNGEN

gann die EZB als erste bereits im August 2007 die Mittel für längerfristige Refinanzierungsgeschäfte mit einer Laufzeit von drei Monaten zu erhöhen; gleichzeitig wurden die Volumina im wöchentlichen Refinanzierungsgeschäft reduziert. Es kam somit zu einer deutlichen Verschiebung der Anteile der Refinanzierung hin zu längeren Fristigkeiten, ohne deren Höhe wesentlich zu ändern. Kurzfristig erhöhte sich immer wieder das Volumen der Forderungen gegenüber den Banken, jedoch wurde diese kurzfristige Liquidität wieder abgezogen. In dieser Phase kam es zu keiner fundamentalen Änderung der Liste der zugelassenen Sicherheiten, auch wurde der Kreis der Banken nicht erweitert, da er, etwa im Vergleich zu den USA, ohnehin schon breit war. Mit dem Zusammenbruch von Lehman Brothers hat sich die Finanzkrise dramatisch verschärft. Bis dahin waren die Liquiditätsmaßnahmen auf die Unterstützung des Interbankmarktes ausgerichtet, auf dessen Funktionieren man grundsätzlich angewiesen war. Nun aber musste die EZB selbst den Ausgleich zwischen den Banken übernehmen, indem sie einerseits Einlagen von Banken mit Liquiditätsüberschuss entgegennahm und andererseits an Banken mit Liquiditätsbedarf gegen Sicherheiten Kredite vergab.

Zur Ausweitung des Refinanzierungsvolumens wurde im Oktober 2008 das Tenderverfahren umgestellt. Bis dahin mussten die Banken bei den Tendern für ein von der EZB festgelegtes Refinanzierungsvolumen bieten. Damit stieg die Unsicherheit für einzelne Banken, ob sie überhaupt eine $\mathrm{Zu}$ teilung erhalten würden. Ab dann wurde das Tenderverfahren auf einen Mengentender umgestellt. Die Liquiditätsbereitstellung sowohl für die wöchentlichen Hauptrefinanzierungs- als auch die längerfristigen Refinanzierungsgeschäfte, deren Laufzeiten weiter ausgeweitet wurden, erfolgte zu einem fixen Zinssatz bei voller Zuteilung. Das Refinanzierungsvolumen ist somit in beiden Bereichen angestiegen, wie in Abbildung 6 ersichtlich ist. Zusätzlich erweiterte die EZB Ende Oktober die Liste der Sicherheiten, die von den Banken für die Liquiditätsbereitstellung beizubringen sind. Ebenso wurde der Zugang zu Liquidität in anderen Währungen, insbesondere geringfügig ausgeweitet hat, wobei dies vor allem durch den steigenden Bargeldbedarf getrieben war. ${ }^{9}$ Angesichts der gestiegenen Nachfrage nach längerfristiger Liquidität und der dadurch ausgelösten Spannungen auf den längerfristigen Geldmärkten be-
9 Die Ausführungen basieren auf der Übersicht über die geldpolitischen Operationen aus den jeweiligen Monatsberichten der EZB. 
in US-Dollar, wesentlich erleichtert. Anfang Mai 2009 kündigte die EZB an, Pfandbriefe (covered bonds) im Umfang von 60 Mrd $€$ anzukaufen. Diese Maßnahme soll dieses für die Finanzierung von Immobilien und Infrastruktur wichtige Marktsegment wieder beleben. Außerdem wurde beschlossen, dass die Europäische Investitionsbank unmittelbar an den Finanzierungsgeschäften der EZB teilnehmen kann. Insgesamt hat die Fed stärker auf unkonventionelle Maßnahmen der Geldpolitik zurückgegriffen als die EZB.

\section{5 \\ Inflationswirkungen nicht-konventioneller Maßnahmen}

Angesichts der Verlängerung der Notenbankbilanzen, die einige der nicht-konventionellen Maßnahmen zur Folge haben, wird in der medialen und wirtschaftspolitischen Debatte gerne das Bild einer Liquiditätsschwemme oder des Anwerfens der Notenpresse bemüht. Dieser Eindruck wurde selbst im ersten Jahr der Krise heraufbeschworen, als die Notenbankbilanzen keine wesentliche Ausweitung erfuhren. Grundsätzlich sagt die Höhe der Notenbankbilanz nichts über den Restriktivitätsoder Expansivitätsgrad der Geldpolitik aus, ebenso wenig über deflatorische oder inflatorische Effekte. Ein wesentlicher Grund dafür ist zunächst, dass der Zusammenhang von monetärer Basis und Geldmenge instabil ist. Eine Ausweitung der Zentralbankgeldmenge, die dazu dient, den durch die Finanzkrise gestiegenen Liquiditäts- bedarf zu decken, geht nicht notwendigerweise mit einem Geldmengenanstieg einher. Dieses Phänomen war in Japan zu Beginn der 2000er Jahre zu beobachten, als das M2-Wachstum stagnierte, obwohl die Zentralbankguthaben stark ausgeweitet wurden. Im Euroraum ging das M3-Wachstum zuletzt trotz Anstiegs der Zentralbankgeldmenge sogar zurück. Darüber hinaus besteht langfristig kein eindeutig gesicherter Zusammenhang von Geldmengenwachstum und Inflation. Eine Ausnahme bilden historische Phasen überaus hoher Inflation. Selbst wenn die Ausweitung der Notenbankbilanz mit höherem Geldmengenwachstum verbunden wäre, lässt sich damit noch keine inflatorische Wirkung gesichert prognostizieren, da die Geldnachfrage zahlreichen strukturellen Einflüssen unterliegt, die sich zudem während einer Finanzkrise laufend ändern können.

Grundsätzlich sind die Wirkungen nicht-konventioneller Maßnahmen schwer vorhersehbar, da zahlreiche Transmissionskanäle gleichzeitig kaum quantifizierbare Effekte entfalten. Zunächst werden das Zinsniveau und die Zinsstruktur beeinflusst, parallel dazu werden Vermögenseffekte wirksam. Inflationserwartungen spielen auch eine erhebliche Rolle. Gilt es, ein Deflationsszenario abzuwenden, so ist die Erhöhung von Inflationserwartungen auf das von der Notenbank bzw. dem Staat gewünschte Niveau sogar intendiert. Wesentlich ist, die Liquidität rechtzeitig zu reduzieren. Der Ausstieg aus den nicht-konventionellen Maßnahmen sollte im Zuge einer nachhaltig einsetzenden Konjunkturbelebung und einer Normalisierung der Bedingungen an den Finanzmärkten erfolgen.



Die ökonomischen Auswirkungen der Krise treffen Europa inzwischen massiv. Die Finanzkrise hat Europa jedoch verzögert und in anderer Weise getroffen, weil sich deren institutionelle Strukturen - vor allem der Finanzmärkte - von denen der USA wesentlich unterscheiden. Die Geldpolitik der EZB hat sich deshalb bisher auf die Liquiditätsbereitstellung für das Bankensystem aus der Perspektive der Finanzmarktstabilität sowie auf die konjunkturelle Steuerung mittels Zinspolitik konzentriert. Die EZB hat ihren Hauptrefinanzierungssatz seit September 2008 von 4,25\% auf $1 \%$ gesenkt. Je mehr sich aber die konjunkturelle Krise in Europa vertiefte und je mehr sich der geldpolitische Zinssatz der Grenze seiner Möglichkeiten annähert, umso mehr verlagert sich der Schwerpunkt $\mathrm{zu}$ unkonventionellen Maßnahmen. Im ersten Jahr der Krise konzentrierten sich diese auf die Liquiditätsversorgung des Finanzsystems, was bis heute fortgesetzt und ausgebaut wurde. Dem europäischen Bankensystem wurde praktisch unbegrenzter Zugang zu Notenbankliquidität eröffnet, um die nach dem Zusammenbruch von Lehman Brothers äußerst angespannte Liquiditätssituation und steigenden Risikoprämien in den Griff zu bekommen. Mit dem Erwerb von Pfandleihbriefen hat die EZB ein Programm aufgelegt, bei dem sie die Refinanzierung der Banken zusätzlich erleichtert und gleichzeitig ein wichtiges Segment im Euroraum-Finanzmarkt belebt. 


\section{LITERATUR}

Bernanke, B. (1999): Japanese Monetary Policy: A Case of Self-Induces Paralysis?, Princeton University Working Paper

Bernanke, B. (2003): Some Thoughts on Monetary Policy in Japan, Speech to the Japan Society of Monetary Economics, Tokyo, May 31, http://www.federalreserve.gov/BoardDocs/Speeches/2003/20030531/ default.htm

Bernanke, B. (2009): The Crisis and the Policy Response, Stamp Lecture, London School of Economics

Bernanke, B./Reinhart, V./Sack, B. (2004): Monetary Policy Alternatives at the Zero Bound: An Empirical Assessment, Brookings Papers on Economic Activity 2, S. 1-78

Bordo, M./Filardo, A. (2005): Deflation and Monetary Policy in a Historical Perspective: Remembering the Past or Being Condemned to Repeat It, in: Economic Policy 44, S. 799-844

Borio, C./Filardo, A. (2004): Back to the future? Assessing the deflation record, Bank for International Settlements, Working Paper 152

Buiter, W. (2009): The wonderful world of negative nominal interest rates, again. VOX, 4 June, http://www.voxeu.org/index.php?q=node/ 3626

Eichengreen, B./O'Rourke, K. (2009): A Tale of Two Depressions, 4 June, http://www.voxeu.org/index.php?q=node/3421

Europäische Zentralbank (EZB) (2009): Ergebnisse der Umfrage zum Kreditgeschäft im Euro-Währungsgebiet vom April 2009, Monatsbericht Mai Fisher, I. (1933): The Debt-Deflation Theory of Great Depressions, in:

Econometrica 1, S. 337-357

FRBSF Economic Letter (2001): Quantitative Easing by the Bank of Japan, November 2
Gerlach, S. (2009): The Risk of Deflation, in: Dewatripoint, M./Freixas, X./ Portes, R.(Hrsg.): Macroeconomic Stability and Financial Regulation: Key Issues for the G20, http://www.voxeu.org/reports/G20 ebook.pdf International Monetary Fund (IMF) (2007): Assessing Global Financial Risks, in: Global Financial Stability Report, April, S. 1-63 International Monetary Fund (IMF) (2008): Stress in Bank Funding Markets and Implications for Monetary Policy, in: Global Financial Stability Report, Oktober, S. 69-103

Jobst, C. (2009): Die Umsetzung der Geldpolitik in der Krise 2007 bis 2008, in: Geldpolitik \& Wirtschaft 1, S. 57-82

Judd, J./Rudebusch, G. (1999): The goals of U. S. monetary policy, in: FRBSF Economic Letter, January 29

Krugman, P. (2005): Is Fiscal Policy Poised for a Comeback?, in: Review of Economic Policy 4, S. 584-597

Minsky, H. (1982): Can "It" Happen Again? Essays on Instability and Finance, New York

Minsky, H. (1986): Stabilizing an unstable economy, Yale

Mishkin, F. (1996): The Channels of Monetary Transmission. Lessons for Monetary Policy, in: NBER Working Paper 5464

Reinhard, C./Rogoff, K. (2009): The Aftermath of Financial Crises, Paper prepared for presentation at the American Economic Association meetings in San Francisco, January 3

Svensson, L. (1997): Inflation Forecast Targeting: Implementing and Monitoring Inflation Targets, in: European Economic Review 6, S. 11111146 